\title{
Efficacy of Abdominal Massage on Feeding Intolerance of Preterm Neonates
}

\author{
Fathia Zaky Mohamed*, Eman Saied Ahmed \\ Pediatric Nursing, Faculty of Nursing, Assiut University \\ *Corresponding author: fath.zaki@yahoo.com
}

Received August 01, 2018; Revised September 03, 2018; Accepted September 12, 2018

\begin{abstract}
Preterm neonates have an increased susceptibility to feeding intolerance which is one of the most significant contributors to their growth failure. Establishing and tolerating adequate enteral nutrition is difficult due to the immaturity of the gastrointestinal system; therefore it is crucial for neonatology nurses to detected this condition as early as possible and use intervention for tackling this problem to improve newborn outcome. Massage is one therapeutic nursing method that can stimulate the parasympathetic activity and induce a more effective response of the digestive system. The aim of this study was to evaluate the efficacy of abdominal massage on feeding intolerance of preterm neonates. A quasi- experimental research design was utilized to meet the aim of this study. Setting This study was conducted in Neonatal Intensive Care Unit (NICU) at Assiut University Children Hospital. Sampling: The study sample comprised all preterm neonates (60 preterm neonates) hospitalized during six months period in Neonatal Intensive Care Unit (NICU) at Assiut University Children Hospital. Three tools were used to collect the required data for this study: - Tool1- An interviewing questionnaire sheet, Tool II - Follow -up sheet and Tool III - Feeding intolerance assessment tool. Results: Highly statistically significant differences were found between the first day and $5^{\text {th }}$ day related to feeding intolerance measurements parameters of the massage group $(\mathrm{P}=0.001)$ and the means of the abdominal circumference, the frequency of vomiting episodes, and the gastric residual volume in the massage group had decreased significantly in the $5^{\text {th }}$ day of the intervention of abdominal massage, as compared with those in the first day and the frequency of defecation and daily weight gain had increased significantly. Concluded that abdominal massage has the potential effect to decrease the gastric residual volume, frequency of vomiting episodes, abdominal distension, and increase the weight and frequency of defecation in enterally fed preterm neonates. The study recommended changing the nursing practice in NICU nurses by emphasizing the importance of applying abdominal massage twice a day as a safe non-invasive intervention as standard of care in enterally fed preterm neonates'.
\end{abstract}

Keywords: preterm infants, abdominal massage, feeding intolerance

Cite This Article: Fathia Zaky Mohamed, and Eman Saied Ahmed, "Efficacy of Abdominal Massage on Feeding Intolerance of Preterm Neonates." American Journal of Nursing Research, vol. 6, no. 6 (2018): 371-379. doi: 10.12691/ajnr-6-6-4.

\section{Introduction}

Worldwide, approximately 15 million births are born preterm each year. It is a major cause of infant morbidity and mortality. [1] Management of the nutritional needs of these premature infants is extremely complex. The combination of gastrointestinal immaturity and high nutrient requirements for catch-up growth predispose these infants to feeding intolerance, which may lead to suboptimal nutrition and subsequent adverse outcomes including reduced brain growth, cognitive delays, and necrotizing enterocolitis (NEC). [2] Furthermore, cesarean delivery, long neonatal intensive care unit (NICU) stay, or antibiotic administration may cause aberrations in intestinal microbial colonization which predispose to feeding intolerance. Given the large and lasting detrimental impact of feeding intolerance on growth and development, it is crucial that this condition be detected as early as possible and treated promptly with evidencebased therapies. Targeted investigation into both treatment and prevention of feeding intolerance should effectively improve preterm infant outcomes. [3]

Goals for preterm infant feeding are to regain the up to $20 \%$ body weight loss experienced in the first week of life and achieve postnatal growth comparable to intrauterine rates of growth and nutrient accretion. Enterally or parenterally fed preterm infants require 120 or $80-100$ $\mathrm{kcal} / \mathrm{kg} / \mathrm{day}$, respectively and up to $4 \mathrm{~g}$ protein $/ \mathrm{kg} /$ day. Meeting these nutritional goals is imperative to achieve desired weight gain up to 2 times that of the term infant improve long-term neurological outcomes, and prevent hospital readmission. [4]

Feeding intolerance is defined as an inability to digest enteral feedings presented as gastric residual volumes (GRV) greater than $50 \%$ of previous feeding volume; abdominal distension or emesis of both; and the disruption 
of the feeding plan (delay, decrease or discontinuation of enteral feeds) precipitated by gastrointestinal signs. Feeding breast milk rather than formula may speed achievement of full enteral feeds, as evidenced by faster achievement of full enteral feeds in VLBW infants fed $\geq 50 \%$ human milk versus who was exclusively formula-fed. Breast milk also offers protection against NEC. [3,5]

Enteral nutrition (EN) is preferable to parenteral nutrition (PN) due its lower costs and avoidance of PN-associated complications, as well as its ability to stimulate gastrointestinal maturation and prevent intestinal atrophy. Anatomical maturation of the gastrointestinal tract is largely complete by 20 weeks gestation, but functional maturation, including motility, gastric acid secretion, gastroesophageal sphincter tone, enzyme activity, and bile acid availability, may not be complete until term gestation. [6]

Enteral Feeding is a major problem in premature infants, resulting in prolonged hospitalization, increased risk of sepsis and a predisposition to serious complications due to prolonged use of parenteral nutrition. The nutritional problems of preterm infants have become particularly relevant on short and long-term development. Vomiting is the most serious complication associated with enteral feeding, increasing the risk of aspiration and pneumonia. Therefore, it is important to know and assess correctly the warning signs of the possible complications of enteral feeding. $[7,8]$

Feeding intolerance prevention strategies are diverse and vary substantially between institutions. Unfortunately, despite best efforts to integrate these strategies, infants may still develop severe feeding intolerance. Nurses will remain critical first-line defenders in detection of feeding intolerance and important advocates for their patients. [3]

Massage is one therapeutic nursing method that can stimulate the parasympathetic activity and induce a more effective response of the digestive system by accelerating peristalsis, decreasing abdominal distension, accelerating the bowel transit time, increasing the frequency of defecation, and decreasing the frequency of vomiting episodes in the daytime. Thus it is consistently associated with increases in vagal activity and gastric motility which in turn, were lead to weight gain. [7,9]

Nursing assessment for early identification of feeding intolerance symptoms has not yet been addressed. It is crucial for neonatology nurses to understand potential physical change they may observe when the preterm infant is experiencing feeding intolerance. Currently, there are no nursing assessment standards of care guidelines that provide focus to feeding intolerance symptoms. However, within the literature, there is noteworthy agreement on the symptoms that are commonly associated with feeding intolerance and of the symptom operational definitions. These components are necessary for developing nursing standard of care guidelines that are designed to encourage judicious reporting of early signs/symptoms of feeding intolerance to health care providers, which, in turn, may improve newborn outcome. [10]

\subsection{Significance of the Study}

Feeding intolerance is one of the most significant contributors to growth failure in preterm infants. The inability to sustain enteral feedings also contributes to extended periods of parenteral nutrition, which often requires central venous access, thereby increasing the risk of infection. Establishing and tolerating adequate enteral nutrition is difficult due to the immaturity of the preterm infants' gastrointestinal system; however, it is important for their normal growth, infection resistance, and longterm cognitive and neurologic development, therefore it is crucial for neonatology nurses to detect this condition as early as possible to improve newborn outcome. [10]

\subsection{Aim of the Study}

The aim of this study was to evaluate the efficacy of abdominal massage on feeding intolerance of preterm neonates.

\subsection{Research Hypothesis}

Preterm neonates who receive abdominal massage were expected to show less feeding intolerance than those who don't.

Preterm neonates who receive abdominal massage would exhibit greater weight gain than those who don't.

A significant relationship would be found between the studied neonates' degree of feeding intolerance and their personal characteristics, medical data and their maternal medical data.

\section{Subjects and Method}

\subsection{Research Design}

Quasi-experimental research design was utilized to meet the aim of this study.

\subsection{Operational definitions}

Feeding intolerance refers to an inability to digest enteral feedings presented as gastric residual volumes (GRV) more than $50 \%$, abdominal distension or emesis of both, and the disruption of the feeding plan. [5]

\subsection{Setting}

This study was conducted in Neonatal Intensive Care Unit (NICU) at Assiut University Children Hospital.

\subsection{Sampling}

The study sample comprised all preterm neonates (60 preterm neonate) hospitalized during six months period in Neonatal Intensive Care Unit (NICU) at Assiut University Children Hospital who met the Inclusion criteria entered the study following approval of their parents and they were randomly assigned to two groups: study group which included (30) preterm neonate receiving abdominal massage therapy and control group which included (30) preterm neonate receiving only routine care of preterm neonates.

\subsection{Inclusion and Exclusion Criteria}

The inclusion criteria included preterm neonates who were being fed by nasogastric tube, gestational age 
(28-36 wks) and birth weight (1000- 2499 gm). Preterm neonates were excluded from this study if they had any contraindication to abdominal massage; intestinal obstruction; major congenital malformation as (congenital heart disease, GIT anomalies); hypoxic injury; respiratory failure with ventilatory support; necrotizing enterocolitis (NEC); Sepsis and any abdominal surgery.

\subsection{Tools of Data Collection}

Three tools were used to collect the required data for this study:-

\section{Tool1- An interviewing questionnaire sheet}

Which was developed by the researcher after reviewing of the related literature to collect relevant information of preterm neonates and their mothers and it includes four parts:

a) -Preterm neonates characteristics as (name, gender, date of admission and diagnosis)

b) - Preterm neonates mother's personal characteristics as (age, educational level and occupation)

c) - Preterm neonates medical data as (Apgar scoring, oxygen therapy, blood transfusion, congenital anomalies and neonatal complications

d)-Preterm neonates' mother's medical data as (antenatal care, type of pregnancy, use of medication during pregnancy, chronic disease, complications during pregnancy, type of delivery and complications during delivery).

\section{Tool II - Follow -up sheet:}

Which was developed by the researcher to measure newborn studied parameters related to feeding intolerance and it include:-

a)-Measurements of studied neonates feeding intolerance criteria as (abdominal circumference, abdominal distention, number of vomiting episodes, gastric residual volume and frequency of defecation).

b) - Daily weight of newborn

\section{Tool III - Feeding intolerance assessment tool:}

It was adapted from A Novel Neonatal Feeding intolerance assessment scoring tool which was developed and validated by Naberhuis, et al., (2016) [11] to assess degree of Feeding intolerance of preterm neonate and risk factors relevant to development of feeding intolerance. It consists of five categories variables including; gestational age at birth, birth weight, newborn feeding substance, newborn postnatal factors and perinatal maternal factors. These 5 categories variables were populated with multiple risk factors, each assigned a numeric point value ranging from 1 to 3 . The numeric score generated upon tool completion, ranging from 1 to 44, reflects the sum of points from each risk factor. The scoring system of this scale was as the following; a score of 1 to 5 was low, from 6 to 8 moderate and 9 or more was high category.

\subsection{Method}

Administrative approval was obtained from the head manger of Assiut University Children Hospital and the head of the Neonatal Intensive Care Unit before implementation of the study.

- Written informed consent was obtained from the parents in both the study and control groups after explaining the aim and nature of the study by the researchers.

- Relevant medical history was gathered from the infant's medical records and their mothers

- A 6 Fr orogastric tube were inserted for these neonates and bolus feedings were given by gravity drainage.

- The gastric residual volume measurement was taken before each feeding administration. A gastric residual volume measurement was made by aspirating with a $5 \mathrm{ml}$ syringe before each feeding. Positioning of the gastric tube was determined by giving $0.5-1 \mathrm{ml}$ of air and listening with a stethoscope at the epigastric region. The GRV measurement was then made. During the GRV measurement, the syringe piston was withdrawn slowly.

- During infant gavage feeding, the flow rate of milk was caused by gravity using the suspension technique without applying the pressure to the injector.

- After placing the nasogastric tube in the study group, abdominal circumference was measured before initiation of the first feeding. Once feedings started, before each feeding meal, follow-up weight, vomiting, GRV measurement, abdominal circumference measurement, abdominal distension measurement (palpation), and frequency of defecation, were assessed at 8:00 am and 2:0 pm. The recorded measurements were daily documented on the "Follow- Up sheet" in this period. Abdominal massage was then applied for 15 minutes one hour before feeding within the 5-day study period.

- Collecting data from control group subjects' neonates in the control group received feedings like the neonates in the massage group.

- Data were collected in the same way from both groups. The control group received the usual care only.

- Abdominal massage was applied to the study group subjects for 15 minutes, 2 times daily, one hour before the subject was fed starting in the 5-day study period. Before applying massage, moisturizing lotion (Baby Lotion) was used for massage application.

\subsection{Abdominal Massage}

The abdominal massage was applied in a clockwise direction over the intestines on the abdominal wall. The following strokes with moderate pressure were applied to the areas of the preterm infants' abdomen:

1 - Place newborn in supine position with head elevated $30^{\circ}-45^{\circ}$

2- Use moisturizing lotion and hold your hand so your pinkie finger's edge can move like a paddle across your baby's belly. Starting at the base of the rib cage, stroke downwards with one hand and then the other in a paddlewheel-like motion.

3- Massage the abdomen with your fingertips in a circular clockwise motion.

4- Do "I Love U" stroke (I on left side, L a cross abdomen along the base of her ribs from her right side to her left and down and $\mathrm{U}$ on right side up and around umbilicus and down left side) 
5- Walk or move your fingers around umbilicus clockwise

6- Hold knee and feet together and gently press the knees up toward abdomen, rotate the baby hips around to the right a few times

7- Place your hand on the tummy (above umbilicus) horizontally and rock your hand from side to side a few times (if cord not completely healed avoid massaging the tummy).

\subsection{Pilot Study}

After developing the tools, a pilot study was conducted on $(10 \%)$ of preterm neonates in neonatal Intensive Care unit (NICU) at Assiut University Children Hospital to test clarity and completeness of the study tools and to determine the time required to fill each tool and they were included in the study sample. According to the results of pilot, the final form was secured before actual study work.

Tool1 \& Tool II were reviewed by a jury of 5 experts in both pediatric nursing and pediatrics fields to test its content validity and the reliability

Tool III was valid and its internal consistency using Cronbach's alpha coefficient method was (0.71).

- Data collection was done five days per week from 8.00 AM to 2.00 PM.

\subsection{Field of the Work}

The study was conducted over a period of six months; from the beginning of November 2017 to the end of April 2018. Data collection was done five days per week from 8.00 AM to 2.00 PM. each intervention take about 30 minutes (15 minutes, 2 times daily) and measurements of studied neonates' parameters related to feeding intolerance criteria take from about 30 to 45 minutes individually for each neonate for about $3-5$ neonates per day. Interviewing the studied neonate's parents was conducted according their available time to collect necessary data. The time needed for each interview ranged from 30 to 45 minutes.

\subsection{Ethical Considerations}

Approval to conduct the study was obtained from the Ethics Committee. After giving the necessary information to the parents who had agreed to participate their neonates in the research, written informed consent was obtained from the parents in both the study group and the control group and they are secured that data will be confidential and used only for the research purpose. The researchers assured voluntary participation, anonymity and confidentiality of each neonate parent who agreed to participate in the study.

\subsection{Statistical Analysis}

Data were verified, coded by the researcher and analyzed using IBM-SPSS 21.0 (IBM-SPSS Inc., Chicago, IL, USA) ${ }^{*}$. Descriptive statistics: Means, standard deviations, medians, ranges and percentages were calculated. Test of significances: chi-square test was used to compare the difference in distribution of frequencies among different groups. For continuous variables; independent t-test analysis was carried out to compare the means. For repeated measurements; paired t-test analysis was carried out to compare the means. A significant $p$ value was considered when it is equal or less than 0.05 .

\section{Results}

Table 1: Shows studied preterm neonate's characteristics and medical data. There were no statistically significant differences between both control group and massage group in terms of gestational age, birth weight, gender and type of feeding. It was found that, average gestational age for both control group and massage group was $(30.70 \pm$ $2.5,29.80 \pm 2.0)$ weeks respectively and the average birth weight was $(1561.0 \pm 461.5,1548.2 \pm 140.7)$ grams respectively. Concerning gender, more than half (55\%) of both groups were males while $(45 \%)$ were females. As regard to type of feeding, it was noticed that the majority $(90 \%)$ of massage group were being fed their mother's milk and formula and only $(10 \%)$ were being fed their mother's milk only while two thirds $(60 \%)$ of the control group were being fed their mother's milk and formula and (40\%) fed their mother's milk only. Regarding medical data of studied preterm neonates, both control and massage groups had low mean $(4.56 \pm 1.5,3.95 \pm 0.9)$ Apgar scoring at the $1 \mathrm{st}$ and 5 th minutes, the majority $(90 \%$, $85 \%$ ) of both groups had oxygen therapy while only $(5 \%)$ of them had minor congenital anomalies respectively.

Table 1. Studied preterm neonates characteristics and medical data

\begin{tabular}{|c|c|c|c|}
\hline Items & $\begin{array}{c}\text { Control Group } \\
(\mathbf{n}=20)\end{array}$ & $\begin{array}{c}\text { Massage Group } \\
(\mathbf{n}=20)\end{array}$ & P-value \\
\hline Gestational age/weeks & $30.70 \pm 2.5$ & $29.80 \pm 2.0$ & $=0.222 *$ \\
\hline Birth weight (g) & $1561.0 \pm 461.5$ & $1548.2 \pm 140.7$ & $=0.911 *$ \\
\hline \multicolumn{4}{|l|}{ Gender } \\
\hline - Female & $9(45 \%)$ & $9(45 \%)$ & $=1.000 * *$ \\
\hline - Male & $11(55 \%)$ & $11(55 \%)$ & \\
\hline \multicolumn{3}{|l|}{ Type of Feeding } & \multirow{3}{*}{$=0.028 * *$} \\
\hline - Mother milk only & $8(40 \%)$ & $2(10 \%)$ & \\
\hline - Mother milk \& formula & $12(60 \%)$ & $18(90 \%)$ & \\
\hline Apgar score & $4.56 \pm 1.5$ & $3.95 \pm 0.9$ & $=0.162 *$ \\
\hline Oxygen therapy & $18(90 \%)$ & $17(85 \%)$ & $=0.322 * *$ \\
\hline Blood transfusion & $6(30 \%)$ & $6(30 \%)$ & $=1.000 * *$ \\
\hline Minor congenital anomalies & $1(5 \%)$ & $1(5 \%)$ & $=1.000 * *$ \\
\hline
\end{tabular}

*T-test was used to compare the mean differences

**Chi-square test was used to compare the percentages between groups. 
Table 2. Studied preterm neonate's maternal personal characteristics and medical data

\begin{tabular}{|c|c|c|c|}
\hline Items & $\begin{array}{c}\text { Control Group } \\
(n=20)\end{array}$ & $\begin{array}{c}\text { Massage Group } \\
(\mathbf{n = 2 0})\end{array}$ & P-value* \\
\hline \multicolumn{4}{|l|}{ Mother's age } \\
\hline$\bullet \leq 30$ years & $13(65 \%)$ & $14(70 \%)$ & $=0.736$ \\
\hline - > 30 years & $7(35 \%)$ & $6(30 \%)$ & \\
\hline \multicolumn{3}{|l|}{ Mother's education } & \multirow{3}{*}{$=0.347$} \\
\hline - Basic education & $17(85 \%)$ & $15(75 \%)$ & \\
\hline - University or higher & $3(15 \%)$ & $5(25 \%)$ & \\
\hline \multicolumn{3}{|l|}{ Mother's occupation } & \multirow{3}{*}{$=1.000$} \\
\hline - Employee & $3(15 \%)$ & $3(15 \%)$ & \\
\hline - Housewife & $17(85 \%)$ & $17(85 \%)$ & \\
\hline Antenatal care & $20(100 \%)$ & $17(85 \%)$ & $=0.115$ \\
\hline \multicolumn{4}{|l|}{ Type of pregnancy } \\
\hline - Single & $17(85 \%)$ & $17(85 \%)$ & $=1.000$ \\
\hline Use of medications during pregnancy & $9(45 \%)$ & $7(35 \%)$ & $=0.519$ \\
\hline Chronic diseases & $6(30 \%)$ & $7(35 \%)$ & $=0.627$ \\
\hline Complications of pregnancy & $13(65 \%)$ & $20(100 \%)$ & $=0.013$ \\
\hline \multicolumn{4}{|l|}{ Type of delivery } \\
\hline - Vaginal & $3(15 \%)$ & $0(0 \%)$ & $=0.115$ \\
\hline - CS & $17(85 \%)$ & $20(100 \%)$ & \\
\hline
\end{tabular}

*Chi-square test was used to compare the percentages between groups.

Table 2: Presents studied preterm neonate's maternal personal characteristics and medical data. No statistically significant differences were found between both control and massage groups regarding all studied preterm neonates mother's personal characteristics and medical data. Regarding mother's personal characteristics, results of the current study revealed that more than two thirds $(65 \%, 70 \%)$ of them in both control and massage groups respectively were in the age group $\leq 30$ years. The majority and most of them $(85 \%, 75 \%$ and $85 \%)$ in both groups respectively had basic levels of education and were housewives. As regard their medical data, it was observed that all $(100 \%)$ of them in the control group and the majority $(85 \%)$ of them in the massage group had antenatal follow up and care during their pregnancy and the majority $(85 \%)$ of them had single pregnancy respectively. Forty five percent $(45 \%)$ of them in the control group and (35\%) in massage group use medications during their pregnancy while about one third $(30 \%, 35 \%)$ of them in both control and massage groups respectively had chronic diseases with complications of pregnancy in all (100\%) of massage group and about two thirds (65\%) of control group. Regarding type of delivery, all (100\%) of them in the massage group and the majority $(85 \%)$ of control group were being delivered by caesarean section.

Table 3: Illustrates comparison between the first day and $5^{\text {th }}$ day means of measurements parameters related to feeding intolerance among both control and massage groups of studied preterm neonate's. Highly statistically significant differences were found between the means of the first day and $5^{\text {th }}$ day related to feeding intolerance measurements parameters of the massage group $(\mathrm{P}=0.001)$ in which gastric residual volume (GRV) was decreased from $(52.78 \pm 9.2)$ in the first day to $(42.38 \pm 3.4)$ in the $5^{\text {th }}$ day while vomiting frequency was decreased from $(1.43 \pm 0.1)$ in the first day to $(0.08 \pm 0.05)$ in the $5^{\text {th }}$ day, defecation frequency was increased from $(0.48 \pm 0.1)$ in the first day to $(1.23 \pm 0.2)$ in the $5^{\text {th }}$ day, abdominal circumference was decreased from $(25.43 \pm 1.7)$ in the first day to $(23.70 \pm 1.3)$ in the $5^{\text {th }}$ day and finally daily weight gain was increased from $(1460.8 \pm 179.6)$ in the first day to $(1545.4 \pm 144.3)$ in the $5^{\text {th }}$ day. Conversely, no statistically significant differences were found between the means of the first day and $5^{\text {th }}$ day of the control group related to gastric residual volume (GRV), vomiting frequency and defecation frequency $(\mathrm{P}=0.057, \mathrm{P}=0.036$ \& $\mathrm{P}=0.349)$ respectively except for abdominal circumference and daily weight gain.

Table 3. Comparison between the first day and $5^{\text {th }}$ day means of measurements parameters related to feeding intolerance among both control and massage groups of studied preterm neonate's

\begin{tabular}{|l|c|c|c|c|c|c|}
\hline \multirow{2}{*}{ Feeding intolerance parameters } & \multicolumn{2}{|c|}{ Control Group } & \multirow{2}{*}{ P-value* } & \multicolumn{2}{|c|}{ Massage Group } & \multirow{2}{*}{ P-value* } \\
\cline { 2 - 6 } & $\mathbf{1}^{\text {st }} \mathbf{d a y}$ & $\mathbf{5}^{\text {th }} \mathbf{d a y}$ & & $\mathbf{1}^{\text {st }}$ day & $\mathbf{5}^{\text {th }}$ day & \\
\hline Gastric residual volume (GRV) & $41.00 \pm 12.8$ & $46.38 \pm 9.9$ & 0.057 & $52.78 \pm 9.2$ & $42.38 \pm 3.4$ & $<\mathbf{0 . 0 0 1}$ \\
\hline Vomiting frequency & $0.75 \pm 0.2$ & $1.13 \pm 0.2$ & $\mathbf{0 . 0 3 6}$ & $1.43 \pm 0.1$ & $0.08 \pm 0.05$ & $<\mathbf{0 . 0 0 1}$ \\
\hline Defecation frequency & $0.73 \pm 0.1$ & $0.60 \pm 0.1$ & 0.349 & $0.48 \pm 0.1$ & $1.23 \pm 0.2$ & $\mathbf{0 . 0 0 1}$ \\
\hline Abdominal circumference (cm) & $25.50 \pm 5.6$ & $27.87 \pm 4.7$ & $<\mathbf{0 . 0 0 1}$ & $25.43 \pm 1.7$ & $23.70 \pm 1.3$ & $<\mathbf{0 . 0 0 1}$ \\
\hline Daily weight gain & $1431.8 \pm 419.7$ & $1561.0 \pm 461.4$ & $<\mathbf{0 . 0 0 1}$ & $1460.8 \pm 179.6$ & $1545.4 \pm 144.3$ & $<\mathbf{0 . 0 0 1}$ \\
\hline
\end{tabular}

Feeding intolerance for the massage and control group.

*Paired T-test was used to compare the mean difference. 
Table 4: Verifies comparison between the mean measurements change about feeding intolerance for the control vs. massage group. Highly statistically significant change $(\mathrm{P}=0.001)$ were found between control and massage groups related to all feeding intolerance measurements except for daily weight gain $(\mathrm{P}=0.149)$. After abdominal massage, GRV changed from $(5.38 \pm 2.6)$ to $(-10.40 \pm 1.6)$, vomiting frequency changed from $(0.38$ $\pm 0.2)$ to $(-1.35 \pm 0.1)$, defecation frequency changed from $(-0.13 \pm 0.1)$ to $(0.75 \pm 0.2)$ and abdominal circumference changed from $(2.37 \pm 0.5)$ to $(-1.73 \pm 0.3)$.

Table 5: displays total mean score and levels of feeding intolerance among control and massage groups of studied preterm neonates. Finding of the present study revealed that total mean score of feeding intolerance was (15.95 \pm 3.2) with a range of $(11$ - 24) in the massage group compared to $(14.65 \pm 3.9)$ with a range of $(8-22)$ in the control group. Regarding levels of feeding intolerance, all $(100 \%)$ of the massage group and the vast majority (95\%) of the control group had high levels of feeding intolerance while only $(5 \%)$ of the control group had moderate levels of feeding intolerance. Nevertheless, there is no one of studied preterm neonates in both control and massage groups had mild levels of feeding intolerance.

Table 4. Comparison between the mean measurements change about feeding intolerance for the control vs. massage group

\begin{tabular}{|l|c|c|c|}
\hline \multirow{2}{*}{ Feeding intolerance parameters } & Control Group & Massage Group & \multirow{2}{*}{ P-value* } \\
\cline { 2 - 4 } & \multicolumn{2}{|c|}{ Change } & $<\mathbf{0 . 0 0 1}$ \\
\hline GRV excess & $5.38 \pm 2.6$ & $-10.40 \pm 1.6$ & $<\mathbf{0 . 0 0 1}$ \\
\hline Vomiting frequency & $0.38 \pm 0.2$ & $-1.35 \pm 0.1$ & $\mathbf{0 . 0 0 1}$ \\
\hline Defecation frequency & $-0.13 \pm 0.1$ & $0.75 \pm 0.2$ & $<\mathbf{0 . 0 0 1}$ \\
\hline Abdominal circumference $(\mathbf{c m})$ & $2.37 \pm 0.5$ & $-1.73 \pm 0.3$ & $=0.149$ \\
\hline Daily weight gain & $129.25 \pm 20.1$ & $84.61 \pm 29.4$ & \\
\hline
\end{tabular}

*T-test was used to compare the mean differences.

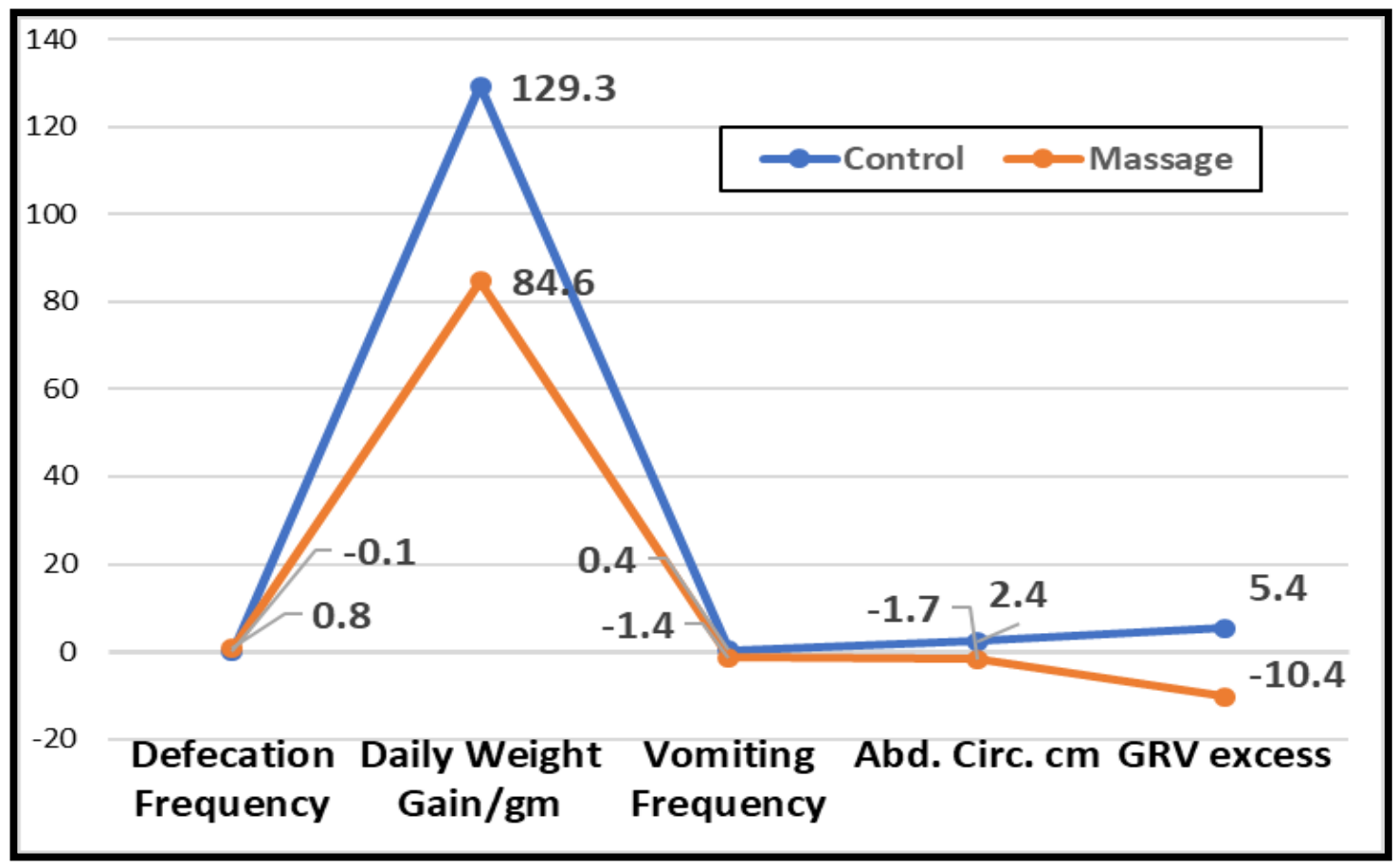

Figure 1. Measurements change about feeding intolerance for the studied groups

Table 5. Total mean Scores and levels of feeding intolerance among control and massage groups of studied preterm neonates

\begin{tabular}{|l|c|c|c|}
\hline Items & $\begin{array}{c}\text { Control Group } \\
(\mathbf{n = 2 0})\end{array}$ & $\begin{array}{c}\text { Massage Group } \\
(\mathbf{n = 2 0})\end{array}$ & P-value \\
\hline Total Score & & $15.95 \pm 3.2$ & $0.251^{*}$ \\
\hline$\bullet$ Mean \pm SD & $14.65 \pm 3.9$ & $15(11-24)$ & \\
\hline$\bullet$ Median (Range) & $14(8-22)$ & & \multirow{2}{*}{$0.500^{* *}$} \\
\hline Scale category (Levels) & & $0(0 \%)$ & \\
\hline$\bullet$ Moderate & $1(5 \%)$ & $20(100 \%)$ & \\
\hline$\bullet$ High & $19(95 \%)$ & & \\
\hline
\end{tabular}

*T-test was used to compare the mean differences

** Chi-square test was used to compare the percentages between groups. 


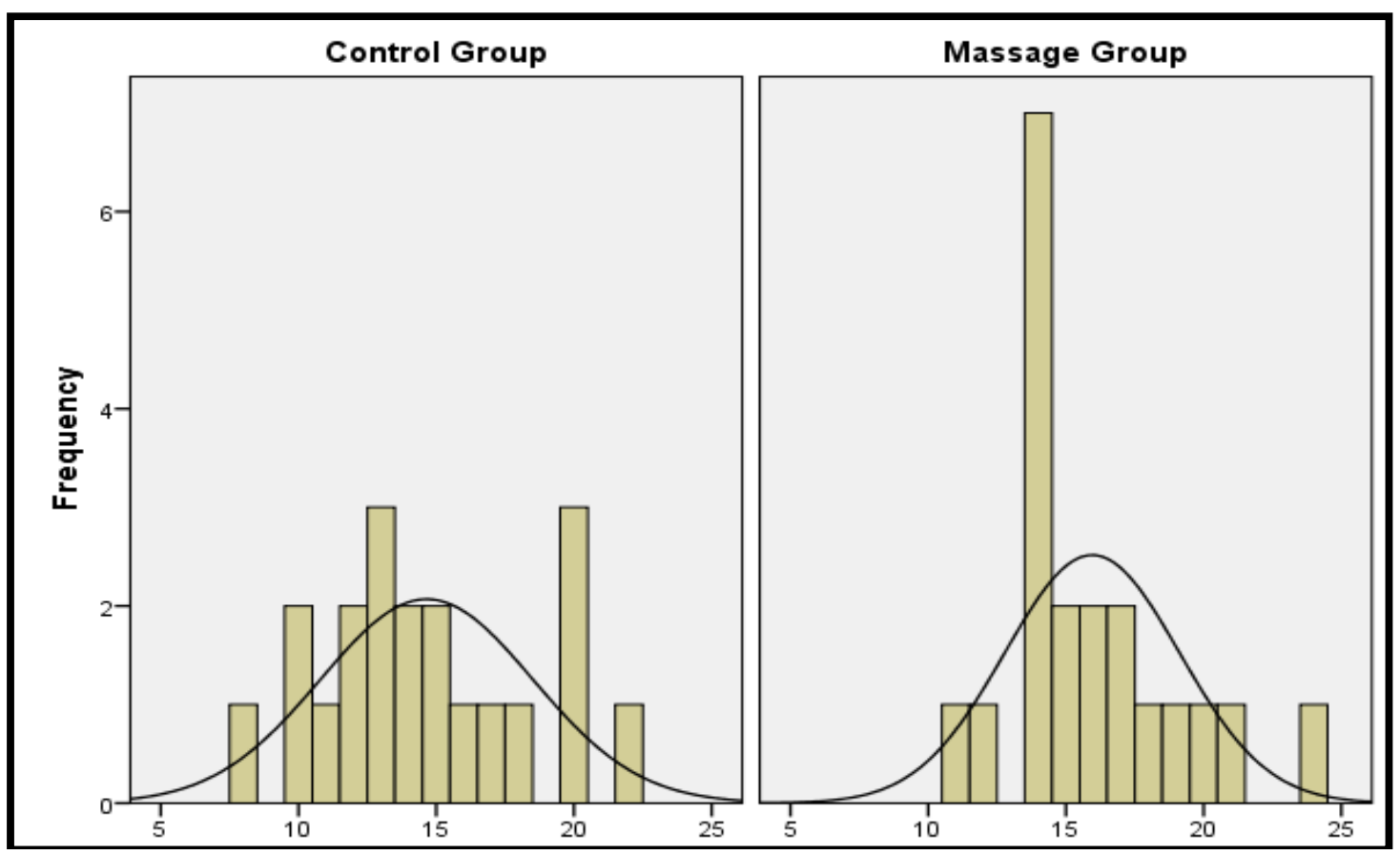

Figure 2. Distribution of feeding intolerance among studied groups

\section{Discussion}

Feeding intolerance is extremely common in premature infants. It is one of the common symptoms seen in the neonatal intensive care unit (NICU) because of digestive-absorptive immaturities of premature infants. The most frequent signs of a suspected feeding intolerance in preterm infants are the presence of gastric residuals and abdominal distension. Delivering proper nutrition can arm this population from developing other co- morbidities. Therefore, it is important to know and assess correctly, the warning signs of the possible complications of enteral feeding. [12,13] Curtailing the occurrence of feeding intolerance has the power to reduce the average premature infants' length of stay in a hospital and costs. [11]

Massage has been described as "a mechanical manipulation of body tissues with rhythmical pressure and stroking for the purpose of promoting health and well-being." It is one of the non-invasive and non-pharmacological interventions for tackling the problem of feeding intolerance in premature infants. There is generally a notion that massage improves circulation and sooth the peripheral and central nervous system. It can stimulate parasympathetic activity resulting in a gastro-intestinal tract (GIT) response that accelerates peristalsis by changing intra-abdominal pressure and creating a mechanical and reflexive effect on the intestines, decreasing abdominal distension and increasing intestinal movements. [7,14]

The present study aimed to evaluate the efficacy of abdominal massage on feeding intolerance of preterm neonates. Results revealed that, there were no statistically significant differences between control and massage group of studied preterm neonates in terms of gestational age, birth weight, gender, type of feeding and their all mother's personal characteristics and medical data. These findings were consistent with Tekgündüz, et al., (2014) [7] who found that there was no statistically significant difference between groups in terms of gestational age, gender, birth weight and type of feeding and also with Diego, et al.,
(2007) [9] who reported that neonatal and maternal demographics and study entry characteristics did not differ between groups.

Concerning gender and type of feeding, more than half of both groups were males and the majority of massage group and two thirds of the control group was being fed their mother's milk and formula. This result was in accordance with Tekgündüz, et al., (2014) [7] who found that $(64.3 \%, 53.8 \% \& 71.4 \%, 84.6 \%)$ of the massage and control groups respectively were males, and fed their mother's milk and formula and apparently agree with Salas, et al. (2015) [15] who determined that the influence of breast milk versus formula had no impact on the time needed to accomplish full feeds. In addition, early postnatal enteral feeding with small amounts of human milk or formula may improve the development of the GI tract, gut hormone release, and gut motility. Minimal enteral feeding has some clinical benefits, such as reducing the time to start full enteral feeding and length of hospitalization without increasing the risk of necrotizing enterocolitis. [16] Consequently, Supporting mothers to express breast milk for their very preterm infants may be one of the most effective and cost-effective interventions. [17]

Interestingly, finding of the current study revealed that, highly statistically significant differences were found between the first day and $5^{\text {th }}$ day related to feeding intolerance measurements parameters of the massage group $(\mathrm{P}=0.001)$ and the means of the abdominal circumference, the frequency of vomiting episodes, and the gastric residual volume in the massage group had decreased significantly in the $5^{\text {th }}$ day of the intervention of abdominal massage, as compared with those in the first day. Moreover, the frequency of defecation and daily weight gain had increased significantly. Conversely, no statistically significant differences were found between the first and $5^{\text {th }}$ day of the control group related to gastric residual volume (GRV), vomiting frequency and defecation frequency $(\mathrm{P}=0.057, \mathrm{P}=0.036 \& \mathrm{P}=0.349)$ respectively 
except for abdominal circumference and daily weight gain. These finding were approximately consistent with Tekgündüz, et al., (2014) [7] in a pre-test, post-test quasi-experimental study to evaluate the efficacy of abdominal massage on feeding intolerance in stable preterm infants fed minimal enteral nutrition. conducted at the NICU of a university hospital in Turkey who found that frequency of vomiting, abdominal circumference and GRV excess of the last day measures decreased to the first day measures for massage group and the difference was found to be statistically significant $(\mathrm{p}<0.05)$ and frequency of defecation, and daily weight gain of the last day measures increased to the first day measures for massage group. In the control group, the differences between the first day and last day of the study were not statistically significant for parameters except for daily weight gain and also go on line with Kaur, et al. (2015) [18] looked at whether gastric residuals or abdominal circumference was a better indicator of feeding intolerance. The results of the study determined that abdominal circumference was superior to gastric residuals in signaling feeding intolerance and with Ameri, et al., (2018) [19] who indicated that gastric residuals, abdominal distention and emesis are regarded as indexes of feeding intolerance and also similar with Diego, et al., (2007) [9] who reported that preterm infants who received a 15 -min massage, three times per day over five days, exhibited greater weight gain than preterm infants receiving standard care alone. In addition, gastricmotility and vagal activity during massage therapy, in turn, were significantly related to weight gain. [20] Furthermore, the increased frequency of defecation has a direct relationship with the decreased abdominal circumference and gastric residual volume. In other words, as the frequency of defecation increases, the abdominal circumference and distension decrease. [21]

Further, the current study revealed that, highly statistically significant change $(\mathrm{P}=0.001)$ were found between control and massage groups related to all feeding intolerance measurements except for daily weight gain $(\mathrm{P}=0.149)$. After abdominal massage, GRV changed from $(5.38 \pm 2.6)$ to $(-10.40 \pm 1.6)$, vomiting frequency changed from $(0.38$ $\pm 0.2)$ to $(-1.35 \pm 0.1)$, defecation frequency changed from $(-0.13 \pm 0.1)$ to $(0.75 \pm 0.2)$ and abdominal circumference changed from $(2.37 \pm 0.5)$ to $(-1.73 \pm 0.3)$. These findings were in agreement with Connolly and Gregory, (2012) [2] who indicated that gastric residuals were the most common reason to delay or change enteral feedings and also similar with Tekgündüz, et al., (2014) [7] who shows that the increase in the number of defecation and the decrease in the number of vomiting instances per day as a result of the decrease in abdominal distension are desired outcomes and also with Carter, (2012) [10] who statement that if a change in abdominal girth is greater than 2 centimeters then that can be a cause for concern. One possible explanation for this result was that abdominal massage is efficient in preventing GRV excess, abdominal distension and vomiting in enterally fed preterm infants.

As regard to, levels of feeding intolerance among control and massage groups of studied preterm neonates. It was observed that, all of the massage group and the vast majority of the control group had high levels of feeding intolerance while only $(5 \%)$ of the control group had moderate levels of feeding intolerance. Nevertheless, there is no one of studied preterm neonates in both control and massage groups had mild levels of feeding intolerance. These results apparently go on line with Kim and Bang, (2018). [12] who reported that feeding intolerance is one of the common symptoms seen in the neonatal intensive care unit (NICU) because of digestive-absorptive immaturities of premature infants and also with Lucchini, et al., (2014) [13] who documented that feeding intolerance is extremely common in premature infants while these results were in contrast with Naberhuis, et al., (2016) [11] who reported that out of sixty-three infants were enrolled in their study, thirteen (7.8\%) infant classified as low risk, $25(15.1 \%)$ as moderate risk, and $128(77.1 \%)$ as high risk and also with Khashana and Moussa,( 2016) [22] who found that the percentage of feeding intolerance in PortSaid, Egypt is $2.6 \%$ of the preterm neonates.

\section{Conclusion}

The present study highlighted the effectiveness of abdominal massage as a safe non-invasive and nonpharmacological intervention and has the potential to decrease the gastric residual volume, frequency of vomiting episodes, abdominal distension, and increase the weight and frequency of defecation in enterally fed preterm neonates. It is therefore possibly appropriate to use this method for preventing, reducing, or eliminating preterm neonates' feeding intolerance.

\section{Recommendations}

In the light of the finding of current study, the following are recommended:-

1- Proper and comprehensive nursing assessment of symptoms of feeding intolerance in preterm neonates' can be instrumental in early identification of this problem and have the potential to dramatically improve infant outcomes.

2- Training programs should be provided for all nurses working in NICUs as regards applying massage therapy and its benefits for premature neonates

3- Changing the nursing practice in NICU nurses by emphasizing the importance of applying abdominal massage twice a day as a safe non-invasive intervention as standard of care helping to prevent GRV excess and abdominal distension in enterally fed preterm neonates'.

4- Encourage mothers to apply abdominal massage therapy pre discharge through teaching sessions and using booklet with colored pictures, hence it can be of additional benefit if performed by a parent or caregiver at home following discharge.

5- Identification of clinical factors that nursing staff can evaluate relevant to feeding intolerance

6- Health care providers should fully inform families prenatally and postnatally of the benefits of human milk

7- Further studies with a larger number of subjects would be conducted to assess the effect of massage therapy on health status of premature neonates 


\section{Acknowledgements}

The researchers thank the mothers for their agreement for applying this study on their premature neonates.

\section{References}

[1] Blencowe, H., Cousens, S., Oestergaard, MZ., Chou, D., Moller, AB., Narwal, R., Adler, A., and et al., (2012): National, regional, and worldwide estimates of preterm birth rates in the year 2010 with time trends since 1990 for selected countries: a systematic analysis and implications. Lancet; 379(9832): 2162-2172

[2] Connolly, T. C., and Gregory, K. E., (2012): Enteral feeding practices in the NICU: Results from a 2009 neonatal enteral feeding survey. Advances in Neonatal Care, 12(1), 46-55.

[3] Naberhuis, J.K., (2015): A multifaceted approach to addressing feeding intolerance in the preterm infant; Dissertation Submitted in partial fulfillment of the requirements for the degree of Doctor of Philosophy in Nutritional Sciences. Graduate College, University of Illinois at Urbana-Champaign, Urbana, Illinois.

[4] Hay, W.W., and Thureen, P., (2010): Protein for preterm infants: how much is needed? How much is enough? How much is too much? Pediatr Neonatol; 51(4): 198-207.

[5] Moore, T., and Wilson, M., (2011): Feeding intolerance: a concept analysis. Adv Neonatal Care; 11: 149-154.

[6] Shulman, R.J., Ou, C.N., and Smith, E.O., (2011): Evaluation of potential factors predicting attainment of full gavage feedings in preterm infants. Neonatology; 99: 38-44.

[7] Tekgündüz, K.Ş., Gürol, A., Apay, S.E., and Caner, I., (2014): Effect of abdomen massage for prevention of feeding intolerance in preterm infants. Turkey; Ital J Pediatr; 40: 89.

[8] Akpinar, H., Uysal, N., and Eşer, I., (2012): The effect of abdominal massage on gastric residual volume: A randomized controlled trial; Gastroenterol Nurs, 35: 117-123.

[9] Diego, M.A., Field, T., Hernandez-Reif, M., Deeds, O., Ascencio, A., and Begert, G., (2007): Preterm infant massage elicits consistent increases in vagal activity and gastric motility that are associated with greater weight gain. Miami, Florida; Acta Paediatr, 96: 1588-1591. ISSN 0803-5253.
[10] Carter, B.M., (2012). Feeding Intolerance in Preterm Infants and Standard of Care Guidelines for Nursing Assessments; Newborn and Infant Nursing Reviews; Volume 12, Issue 4, P. 187-201.

[11] Naberhuis, J., Wetzel, C., and Tappenden, K. A., (2016). A novel neonatal feeding intolerance and necrotizing enterocolitis risk-scoring tool is easy to use and valued by nursing staff; Advances in Neonatal Care, 16 (3), 239-244.

[12] Kim, HY., and Bang, KS., (2018): The effects of enteral feeding improvement massage on premature infants: A randomized controlled trial; J Clin Nurs. 27: 92-101.

[13] Lucchini, R., Bizzarri, B., Giampietro, S., and De Curtis, M., (2014): Feeding intolerance in preterm infants. How to understand the warning signs; J Matern Foetal Neonatal Med, 24:72-74.

[14] Rad, Z.A., Haghshenas, M., Javadian, Y., Hajiahmadi, M., and Kazemian, F., (2016): The effect of massage on weight gain in very low birth weight neonates; J Clin Neonatol; 5: 96-9.

[15] Salas, A. A., Cuna, A., Bhat, R., McGwin, G., Carlo, W. A., and Ambalavanan, N., (2015): A randomized trial of re-feeding gastric residuals in preterm infants; Archives of Disease in Childhood. Fetal \& Neonatal Edition, 100, F224-F228

[16] Fanaro, S., (2012). Strategies to improve feeding tolerance in preterm infants; J Maternal Fetal Neonatal Med, 25: 54-56.

[17] McGuire, W., Young, L., and Morgan, J., (2015): Preventing necrotizing enterocolitis in very preterm infants: Current evidence; Pediatrics and Child Health, 25 (7), 265-270.

[18] Kaur, A., Kler, N., Saluja, S., Modi, M., Soni, A., Thakur, A., and Garg, P., (2015): Abdominal circumference or gastric residual volume as measure of feed intolerance in VLBW infants; Journal of Pediatric Gastroenterology and Nutrition, 60 (2), 259-263.

[19] Ameri, GF., Rostami, S., Baniasadi2,H., Pour Aboli , B and Ghorbani, F., (2018): The Effect of Prone Position on Gastric Residuals in Preterm Infants ;Journal of Pharmaceutical Research International (JPRI), 22(2): 1-6,; Article no.JPRI. 40433.

[20] Field, T., Diego, M., and Hernandez-Reif, M., (2011). Potential underlying mechanisms for greater weight gain in massaged preterm infants; Infant Behav Dev, 34: 383-389.

[21] Shaeri, M., Ghadami, A., Valiani, M., Armanian, AM., Amini Rarani, S., (2017). Effects of Abdominal Massage on Feeding Tolerance in Preterm Infants Hospitalized in Selected Hospitals of Isfahan- Iran; Int J Pediatr; 5(3): 4503-10.

[22] Khashana, A., and Moussa, R., (2016). Incidence of feeding intolerance in preterm neonates in neonatal intensive care units, Port Said, Egypt. J Clin Neonatol; 5: 230-2. 